\title{
COMPUTER AIDED DESIGN OF ADJUSTABLE THIN FILM RESISTORS
}

\author{
MARCEL FRANCOIS \\ M.B.L.E., Tweestationsstraat, 80, 1070 Brussel \\ (Received June 11, 1979)
}

\begin{abstract}
A computer program has been developed, which calculates any adjustable thin film resistor from simple input data (electrical, geometrical, positional, orientational), punched on "input cards". The "output cards", which contain the coordinates, are fed to a numerically controlled draughting machine, which produces either an intermediate ink-on-paper drawing or the final cut-and-strip artwork. Our CAD method proceeds in several steps, offering all the permitted solutions and providing for designer's options.
\end{abstract}

\section{INTRODUCTION}

The design of a thin film hybrid integrated circuit presents some particular features:

As compared to printed circuit boards:

1) We have integrated thin film resistors, which must be adjustable "in situ" and must be designed individually.

2) The whole conductor-resistor network must lie in the same plane.

As compared to thick film integrated circuits:

1) All the resistors of a circuit have the same sheet resistance, so that different resistances can only be obtained by different geometries. On a same circuit the number of squares may vary between 10 and 10.000 .

2) The sheet resistance has a batch spread of typically $\pm 20 \%$.

\section{ADJUSTMENT OR TRIMMING}

At present most of the manufacturers adjust their thin film resistors individually by means of a programmable laser facility and so do we. We shall refer to it as the "trim-laser".

Adjustment, usually called "trimming", may be either continuous or discrete, either predictive or sequential, either pure or mixed.

1) Continuous trimming is accomplished by making a blind cut into the resistor body (Figure 1) or into a protruding part (Figures 2 and 3), usually called a "flag".

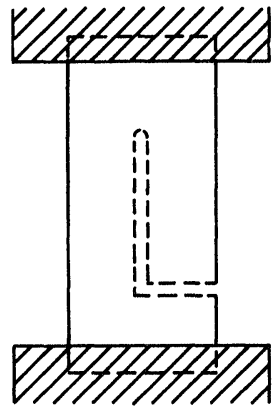

FIGURE 1 Continuous trimming by cutting into the body.

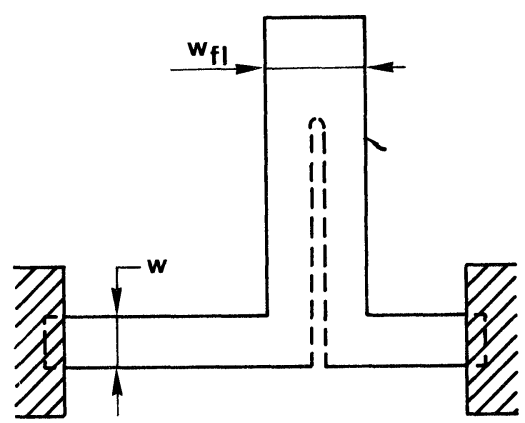

FIGURE 2 Continuous trimming by cutting into a flag.

The trim-laser monitor measures the resistance continuously while the laser beam moves, and cuts the beam energy when the resistor comes within tolerance.

2) Discrete trimming. The resistor consists of or is provided with electrically parallel paths. In the case 


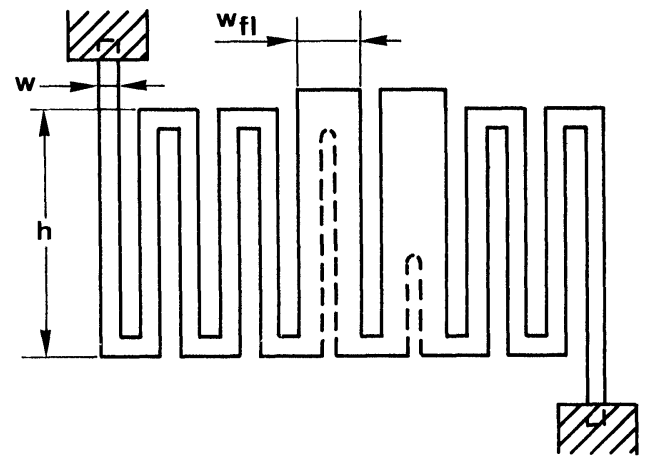

FIGURE 3 Continuous trimming.

of a meander this is usually accomplished by shorting adjoining legs to form a "loop" (Figures 4 and 5). Trimming is done by cutting through some of these parallel paths, i.e. in the case of a meander by opening one or more loops.

3) Predictive trimming. The trim-laser monitor measures the resistance once before trimming, and based on that measurement "decides" how many loops will be opened (Figure 4).

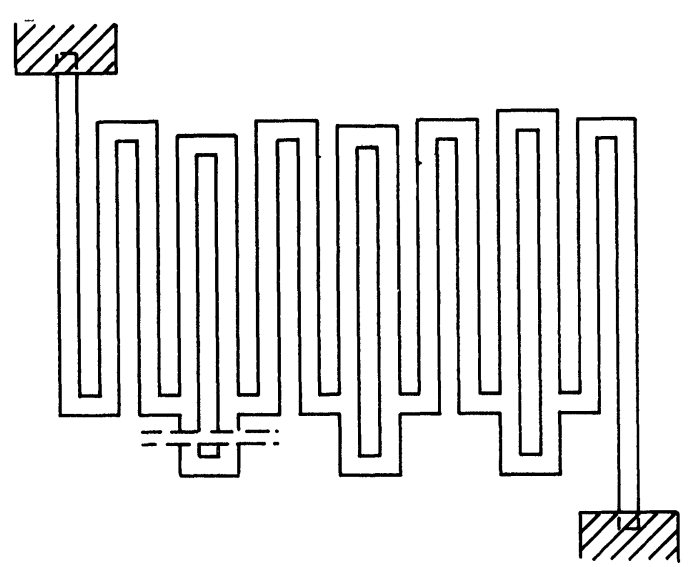

FIGURE 4 Discrete trimming by opening a loop.

4) Sequential trimming. For each loop a measurement is made to "decide" whether it will be opened or not.

5) Mixed trimming. All the preceding types of trimming can be combined. In this paper the phrase will only refer to the combination of predictivediscrete and continuous trimming (Figure 5).

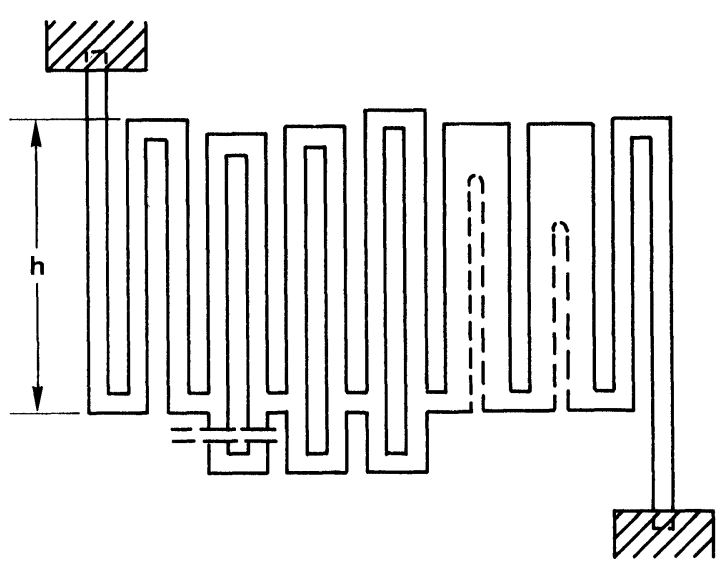

FIGURE 5 Mixed trimming.

\section{DESIGN OF ADJUSTABLE RESISTORS}

\subsection{Symbols. Outline of the resistor before trimming}

$$
\begin{aligned}
R, R_{n} & =\text { actual resp. nominal value of } \\
& \text { resistance } \\
\pm \Delta R, \pm \delta R= & \text { tolerance of resistance } \\
R_{S}, R_{S n} & =\text { actual resp. nominal value of sheet } \\
& \text { resistance } \\
\pm \Delta R_{S}, \pm \delta R_{S}= & \text { admitted range of sheet resistance } \\
& \text { values } \\
N, N_{n} & =\text { actual resp. nominal value of number } \\
& \text { of squares. }
\end{aligned}
$$

Since any kind of trimming increases the resistance, the outline of the resistor before trimming must be such, that the resistance has the highest acceptable value for the highest admitted sheet resistance.

Therefore its number of squares must be

$$
N_{0}=\frac{R_{n}+\Delta R}{R_{S n}+\Delta R_{S}}=\frac{1+\delta R}{1+\delta R_{S}} N_{n}
$$

\subsection{Continuous trimming}

At maximum adjustment, which occurs at minimum $R_{S}$, the number of squares must be

$$
N_{1}=\frac{R_{n}-\Delta R}{R_{S n}-\Delta R_{S}}=\frac{1-\delta R}{1-\delta R_{S}} N_{n}
$$

In addition to Eq. (1) and Eq. (2) there are further 
requirements:

a) The resistor must withstand dissipation at any stage of the trim operation.

b) We must take account of the cut sensitivity. The dimensions must be large enough to avoid overshooting.

c) The dimensions of the rectangle and the width of the flag must be large enough to allow for trimming errors.

\subsection{Predictive trimming}

The admitted range of sheet resistance values $R_{S n} \pm \Delta R_{S}$ is subdivided into "domains" (Figure 6), whose boundaries are denoted by $R_{S 0}, R_{S 1}, R_{S 2}$, etc. Within the respective domains the meander must have the number of squares $N_{0}, N_{1}, N_{2}$, etc., corresponding to $0,1,2$, etc. loops to be cut.

Clearly, the loops must be dimensioned so that, going from right to left, within each domain the resistance varies between $R_{n}+\Delta R$ and $R_{n}-\Delta R$, and, when jumping from one domain to the next, the resistance jumps back from $R_{n}-\Delta R$ to $R_{n}+\Delta R$. It can be shown that both the $N_{i}$ and the $R_{S i}$ form a geometric series with ratio $f$ and $1 / f$ respectively, $f$ being given by

$$
f=\frac{R_{n}+\Delta R}{R_{n}-\Delta R}=\frac{1+\delta R}{1-\delta R}
$$

Now, instead of the conventional tolerances $20 \%$, $10 \%, 5 \%$ we use tolerances which are naturally derived form 1, 2, 3, 4 loops. Obviously, these "predictive tolerances" are obtained by subdividing
TABLE I

Predictive tolerances

\begin{tabular}{lccl}
\hline dom & $\delta R$ & $\delta R_{g}$ & loops \\
\hline 2 & $10,10 \%$ & $10,9 \%$ & 1 \\
3 & 6,75 & 7,5 & 2 \\
4 & 5,06 & 5,8 & 3 \\
5 & 4,05 & 4,8 & 4 \\
\hline
\end{tabular}

the range $-\Delta R_{S},+\Delta R_{S}$ into respectively $2,3,4,5$ domains (Figure 6). Since the $R_{S i}$ form a geometric series, we have

$$
f=\left(\frac{R_{S n}+\Delta R_{S}}{R_{S n}-\Delta R_{S}}\right)^{1 / \text { dom }}=\left(\frac{1+\delta R_{S}}{1-\delta R_{S}}\right)^{1 / \text { dom }}
$$

where dom $=$ number of domains.

From Eq. (3) we deduce easily

$$
\delta R=\frac{f-1}{f+1}
$$

As an example we show in Table I the "predictive tolerances" corresponding to $\delta R_{S}=20 \%$. Introducing a safety margin we denote by $\delta R_{g}$ the "guaranteed tolerance". In this case $\delta R_{g}=\delta R+0.75 \%$. These "predictive tolerances" often enable the saving of a loop.

\section{GENERAL METHOD OF OUR C.A.D.}

Our C.A.D. proceeds in several steps. At each step the designer makes certain choices (options) and
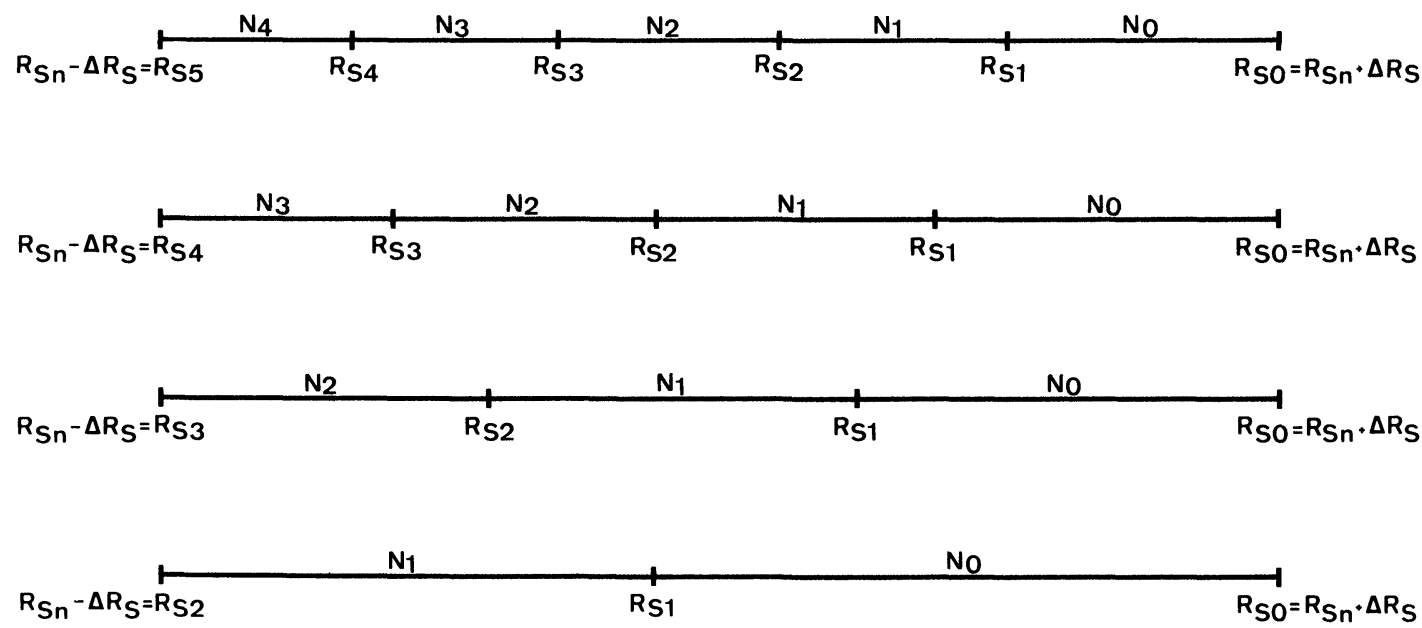

FIGURE 6 Sheet resistance domains. 
puts them into the computer together with fixed requirements. He gets back more information, which enables him to make further choices.

We have built up a FORTRAN computer program, which calculates and draws any thin film resistor from simple input data. The input data are punched on cards, which we shall refer to as the "input cards". There is one card (possibly 2 or 3 ) for the circuit as a whole and one card (possibly 2 or 3 ) for each circuit element.

Some results are only printed on paper, others are also punched on cards, which we shall refer to as the "output cards". These output cards are fed to a numerically controlled draughting machine, which produces an ink-on-paper drawing to enable the results to be visualized. At the last step the same cards are fed to the same (or similar) machine, where the pen is replaced by a chisel and the paper by a two-foil mylar sheet. This machine then produces the cut-and-strip artwork.

Instead of output cards one can use any other medium such as punched tape, magnetic tape or magnetic disc, and the cut-and-strip machine can be replaced by a photoplotter.

\section{NATURE OF THE INPUT DATA. CLASSIFICATION OF CIRCUIT ELEMENTS}

The input data are of electrical, geometrical, positional and orientational nature. First of all, we divide the circuit elements into 5 classes, each referenced by a two-letter symbol: standard conductor patterns $(C \varnothing)$, rectangular resistors (RE), meander resistors (ME), interdigited resistors (DI), locally constructed conductors (L $\emptyset$ ). The classes are divided into subclasses referenced by a number. With regard to geometry we distinguish the general aspect, identified by class and subclass, and the geometrical details, or shape. In order to specify the geometrical, positional and orientational data we set up a "classification list", which gives all necessary definitions.

For every circuit element the following data are given: reference number (i), class and subclass (ge $\emptyset \mathrm{m})$, orientation $(\mathrm{mx}, \mathrm{my}, \mathrm{r} \varphi)$, position $(\mathrm{xb}, \mathrm{yb})$.

For a standard conductor pattern only one more datum (n) suffices to complete the specification.

For every resistor the following data are added: nominal value of resistance $(\mathrm{Rn})$, guaranteed tolerance $(\mathrm{tg})$, number of $\mathrm{R}_{\mathrm{S}}$ - domains (døm), power $(\mathrm{P})$, thermal spreading factor $(\mathrm{g})$.
Further, more data can be added to detail the geometry: length (1), width (w), end point (xe, ye), position of flag (xfl), lower and upper limit of meander (yl $\phi$, yup), number of flags (nf), total number of legs (lg), number of legs before first loop (lgl), between successive loops (lgb), between last loop and flags $(\lg 3)$, distances to edge sensing resistor (desr1, desr2).

\section{CONSECUTIVE STEPS IN OUR C.A.D.}

\subsection{First Step}

Without C.A.D. a meander resistor is very complicated to design. In most cases it can be drawn in several ways, it is difficult to predict which geometry has to be chosen, whether this geometry will satisfy the design rules, what will be its area. This first step gives the answer.

Only class and subclass and the electrical characteristics are put in, e.g.

$\begin{array}{llllllll}\mathrm{i} & \text { ge } \phi \mathrm{m} & \mathrm{Rn} & \mathrm{tg} & \mathrm{d} \phi \mathrm{m} & \mathrm{P}(\mathrm{W}) & \mathrm{g} & \mathrm{w} \\ 120 & \text { ME05 } & 150000 . & - & 4 & 0.001 & 1.0 & -\end{array}$

where $\mid$ ME05 stands for a meander with loops. Then the computer program calculates all the possible meanders of the ME05 type that satisfy the given data and the design rules (i.e. opposite ends, minimum distance to connections, $\mathrm{h} / \mathrm{w} \geqslant 6$, electric field between adjoining legs $\leqslant \mathrm{E} \max$, etc.), and prints out a table giving the number of legs, the dimensions of loops and flags, total height and breadth of the bulk part.

At the end of the first step the designer makes a choice from the offered possibilities.

\subsection{Second Step}

Having replaced every pattern by a simple figure the designer finds an arrangement. Good use can be made of one of the available graphic systems with screen display. Then he introduces the standard conductor patterns, the rectangular, meander and interdigited resistors. For every element he specifies class and subclass, orientation and position; for the resistors he adds the electrical data and possibly w or 1 ; for the meanders he further adds the number of legs. So, e.g.

$\begin{array}{lllllcccc}\mathrm{i} & \text { ge } \phi \mathrm{m} & & \mathrm{Rn} & \mathrm{tg} & \mathrm{d} \phi \mathrm{m} & \mathrm{P}(\mathrm{W}) & \mathrm{g} & \mathrm{w} \\ 310 & \mathrm{ME07} & & 120000 . & 0.01 & 5 & 0.008 & 1.0 & - \\ & \mathrm{mx} & \mathrm{my} & \mathrm{r} \phi & \mathrm{xb} & \mathrm{yb} & \mathrm{lg} & & \\ & \mathrm{f} & \mathrm{t} & \mathrm{f} & 128 . & 84 . & 15 & & \end{array}$

Now the computer program not only calculates and prints the dimensions but also the coordinates 
of every point of every circuit element. In addition these coordinates are punched on cards. For the designer's ease these output cards are furnished in three packs, one for the conductors, one for the resistors and one for the mechanical data (i.e. substrate and components). With these output cards the automatic draughting machine then makes a three-colour-drawing.

\subsection{Third Step}

Inspecting this drawing the designer

a) makes positional and orientational corrections,

b) adds detailed geometrical data such as: number of flags (nf), position of loops and flags $(\lg 1, \operatorname{lgb}, \lg 3)$, position of end point (xe, ye), lower and upper limit of bulk part of meander (ylф, yup), etc.

c) introduces the "edge sensing resistor", which is necessary for the alignment of the thin film circuit with the laser coordinate system. An automatic ink-on-paper drawing is made.

\subsection{Fourth Step}

The designer constructs the conductor connections. Any broken line is generated by simply writing the coordinates of its successive points. However, for connectors consisting merely of $\mathrm{x}$ - and $\mathrm{y}$ - lines, only one coordinate per line has to be introduced. An automatic ink-on-paper drawing is made.

\subsection{Fifth Step}

When the designer is completely satisfied with the last ink-on-paper drawing, he uses the same output cards, more exactly two packs of the three, to make the cut-and-strip artwork.

\section{ADVANTAGES}

1) Saving of time. Any resistor can be drawn at once from simple input data and can be adapted to its surroundings. Moreover, for a meander all possible solutions are presented.

2) A number of different layouts can be generated simultaneously by changing a few cards, and then offered for discussion.

3) Minimum area. Each resistor can be drawn at once within the smallest area.

4) Better performance. For each resistor the best shape can be chosen, especially for meanders: no superfluous bends, all legs of the same length.

5) Accuracy. While on a digitizer all dimensions are rounded off to $1 \mathrm{~mm}$ on the drawing, we now have a numerical accuracy of $0,01 \mathrm{~mm}$.

6) Calculation errors are avoided.

7) Corrections and modifications are easily made by changing a few cards.

\section{APPLICATIONS}

A number of hybrid thin film circuits have been worked out by this method. 

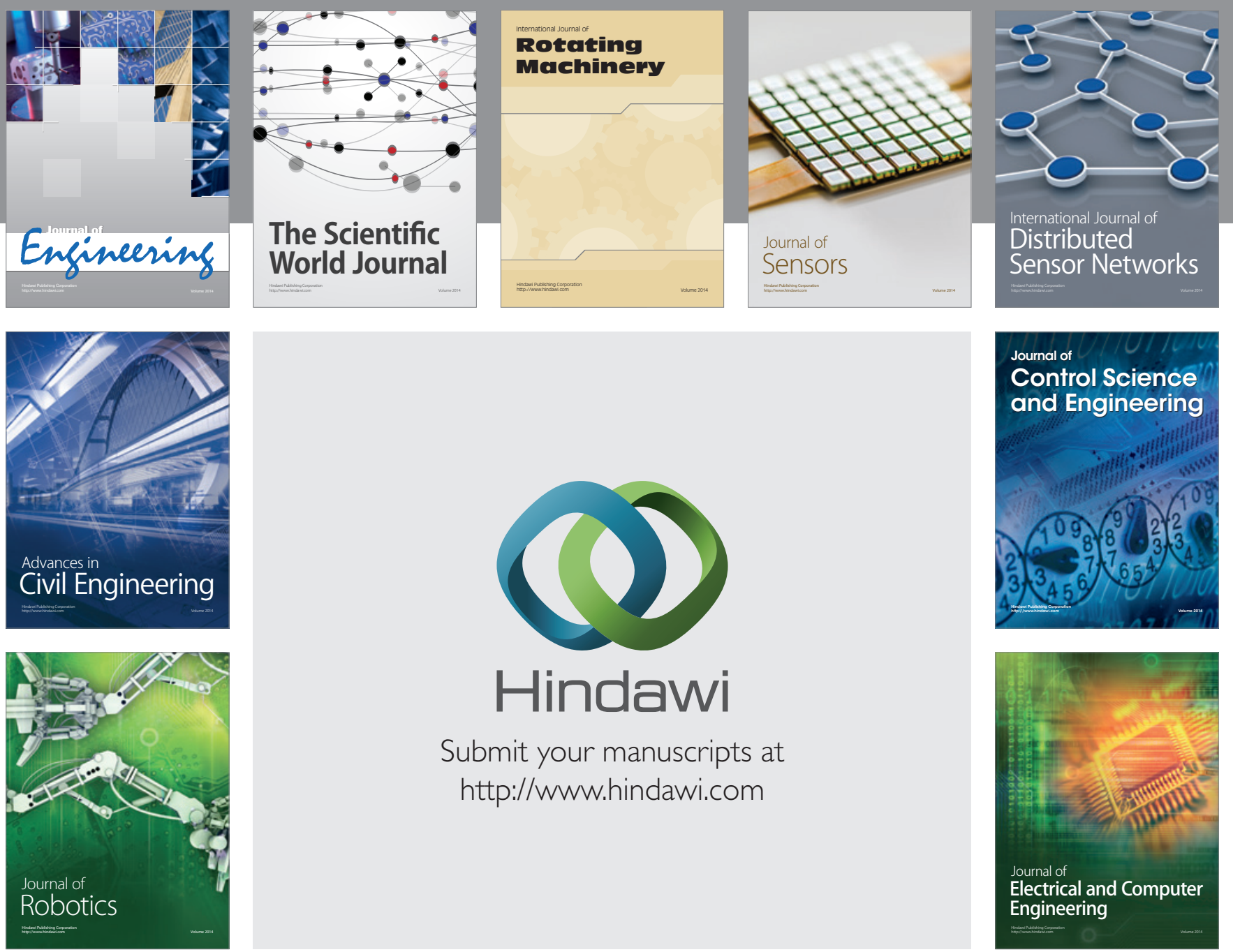

Submit your manuscripts at

http://www.hindawi.com
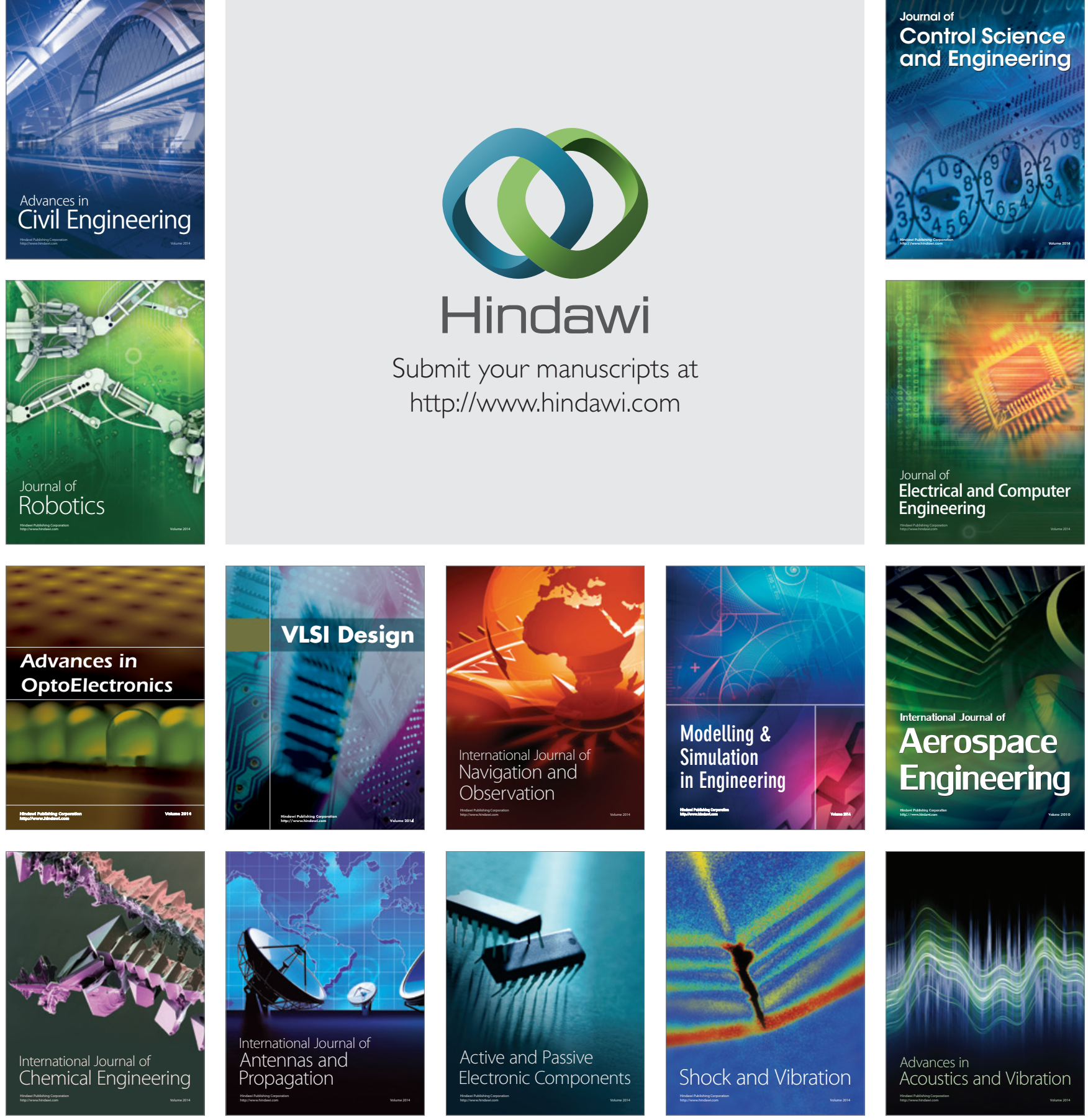